\title{
Activation of the intrinsic mitochondrial apoptotic pathway in swine influenza virus-mediated cell death
}

\author{
Young Ki Choi ${ }^{1}$, Tae-Kyung Kim ${ }^{2}$, \\ Chul-Joong $\mathrm{Kim}^{3}$, Joong-Seob $\mathrm{Lee}^{2}$, \\ Se-Young $\mathrm{Oh}^{2}$, Han Soo Joo ${ }^{4}$, \\ Douglas N. Foster ${ }^{5}$, Ki-Chang Hong ${ }^{2}$, \\ Seungkwon $\mathrm{You}^{2}$ and Hyunggee $\mathrm{Kim}^{2,6}$ \\ ${ }^{1}$ College of Medicine and Medical Research Institute \\ Chungbuk National University \\ Cheongju 361-711, Korea \\ ${ }^{2}$ The Laboratory of Cell Growth and Function Regulation \\ Division of Bioscience and Technology \\ College of Life and Environmental Sciences \\ Korea University \\ Seoul 136-701, Korea \\ ${ }^{3}$ College of Veterinary Medicine \\ Chungnam National University \\ Yusung, Daejeon 305-764, Korea \\ ${ }^{4}$ Department of Veterinary Medicine \\ ${ }^{5}$ Department of Animal Science \\ University of Minnesota, St. Paul, MN, USA \\ ${ }^{6}$ Corresponding author: Tel, 82-2-3290-3059; \\ Fax, 82-2-3290-3475; E-mail, hg-kim@korea.ac.kr
}

\section{Accepted 30 November 2005}

Abbreviations: CPE, cytopathic effects; MDCK, Madin-Darby canine kidney cell; PK-15, porcine kidney-15 cell; SIV, swine influenza virus; ST, swine testicle cell; TCID $_{50}$, tissue culture infectious dose 50

\begin{abstract}
The mitochondrial pathway of swine influenza virus (SIV)-induced apoptosis was investigated using porcine kidney (PK-15) cells, swine testicle (ST) cells, and HeLa cervical carcinoma cells which are known not to support viral replication. As judged by cell morphology, annexin V staining, and DNA fragmen tation, PK-15 and ST cells infected with three different subtypes of SIV (H1N1, H3N2, and H1N2) were obviously killed by apoptosis, not necrosis. SIV infection in PK-15 and HeLa cells was shown to decrease the cellular levels of $\mathrm{Bcl}-2$ protein com pared to that of mock-infected control cells at $24 \mathrm{~h}$ post-infection, whereas expression levels of Bax protein increased in the PK-15 cells, but did not increase in HeLa cells by SIV infection. Cytochrome
\end{abstract}

c upregulation was also observed in cytosolic fractions of the PK-15 and HeLa cells infected with SIV. Apoptosome (a multi-protein complex consisting of cytochrome c, Apaf-1, caspase-9, and ATP) formation was confirmed by immunoprecipitation using cytochrome $c$ antibody. Furthermore, SIV infection increased the cellular levels of TAJ, an activator of the JNK- stressing pathway, and the c-Jun protein in the PK-15 and HeLa cells. Taken together, these results suggest that the mitochondrial pathway should be implicated in the apoptosis of PK-15 cells induced by SIV infection.

Keywords: apoptosis; cytochrome c; influenza virus; mitochondria; proto-oncogene proteins $\mathrm{c}-\mathrm{Bcl}-2$; proto-oncogene proteins $\mathrm{c}$-jun

\section{Introduction}

Programmed cell death, or apoptosis, is central to many physiological processes, including tissue atrophy, development of the immune system, and tumor biology (Majno and Joris, 1995; Jacobson et al., 1997). Apoptosis also plays an important role in the pathogenesis of many infectious diseases, including those caused by viruses (Young et al., 1997; Ludwig et al., 1999; Roulston et al., 1999). Many viral infections provoke apoptosis in host cells, but several viruses have also been implicated in the inhibition of apoptosis (Teodoro and Branton, 1997). Although there is no obvious advantage for apoptosis induction by a cytopathogenic virus, the influenza virus induces apoptosis in numerous cell types, both in vivo (Mori et al., 1995) and in vitro (Morris et al., 1999; SchultzCherry et al., 2001).

Two primary pathways induce apoptosis in mammalian cells (Hengartner, 2000). First, the death-receptor pathway is trigged by members of the death receptor superfamily (such as CD95 and TNFRI). Binding of CD95 ligand to CD95 induces receptor clustering and formation of a death-inducing signal complex. This complex recruits multiple procaspase8 molecules into close proximity via the adaptor molecule FADD (fas-associated death domain protein), which results in caspase-8 activation through proximity. Caspase- 8 activation can be blocked by recruitment of the degenerated caspase homologue c-FLIP. The second apoptosis pathway is induced by mitochondria in response to extracellular cues 
and internal insults, such as DNA damage (Rich et al., 2000). These diverse response pathways converge on mitochondria, often through the activation of a pro-apoptotic member of the Bcl-2 family, such as Bax, Bad, Bim, and Bid. Pro- and anti-apoptotic Bcl-2 family members meet at the surface of mitochondria, where they compete to regulate the exit of cytochrome $c$ by a mechanism that is still disputed. If the pro-apoptotic camp wins, an array of molecules is released from the mitochondrial compartment. Principal among these released molecules is cytochrome c, which associates with Apaf-1 and then casepase- 9 to form the apoptosome. Activation of caspase- 8 and -9 initiates the activation of a cascade of caspases (aspartate-specific cysteine proteases), including caspase-3 (Kumar, 1999). Caspase3 activities have been shown to control both the death-receptor and the mitochondrial pathways (Zheng et al., 1998). In the swine population, three predominant subtypes are prevalent; the classical swine $\mathrm{H} 1 \mathrm{~N} 1$ which is genetically and antigenically similar to the human $\mathrm{H} 1 \mathrm{~N} 1$ virus, the H3N2 "human-like" virus, and a reassortant H1N2 swine virus containing $\mathrm{HA}$ of classical $\mathrm{H} 1 \mathrm{~N} 1$ and other genes (NA) of "human-like" swine H3N2 virus (Choi et al., 2002).

Influenza viruses induce apoptosis in cells that are permissive of viral replication (Morris et al., 1999), as well as cells which do not support viral replication, such as HeLa cells and lymphocytes. Both cellular and viral factors are involved in this process, which may also depend on the cell type. Although influenza virus-induced apoptosis is inhibited by $\mathrm{Bcl}-2$ (Olsen et al., 1996), V-FLIP, and crmA (Takizawa et al., 1999), the best characterized pathway of influenzainduced apoptosis is that activation of receptor-mediated signaling is associated with the activation of caspase-8 (death receptor pathway) (Takizawa et al., 1995; Fujimoto et al., 1998). However, although the $\mathrm{Bcl}-2$ family is associated with apoptosis in various viral infections (Conti et al., 1998; Duncan et al., 1999; Lewis et al., 1999), there have been few reports regarding the activation of members of the Bcl-2 family by the influenza virus. Hence, we examined expression mitochondrial pathway-associated gene regulation in the porcine kidney cell line (PK-15) and HeLa cells infected with three subtypes of swine influenza viruses (SIV).

\section{Materials and Methods}

\section{Cells and viruses}

The porcine kidney epithelial (PK-15) cell line and swine testicle (ST) cell line were purchased from American Type Culture Collection (ATCC) and main- tained in Minimum Essential Medium (MEM) containing $5 \%$ fetal bovine serum (FBS), $2 \mathrm{mM}$ glutamine, and $1 \%$ penicillin/streptomycin at $37^{\circ} \mathrm{C}$. HeLa cells were maintained in Dulbecco's Modified Eagle Medium (DMEM) containing 5\% FBS, $2 \mathrm{mM}$ glutamine, and $1 \%$ penicillin/streptomycin at $37^{\circ} \mathrm{C}$. All cell culture reagents were purchased from GibcoBRL. Swine influenza viruses $[A / S w i n e / N e w$ Jersey/11/76 (H1N1), A/SW/MN-1480/00 (H1N2), and A/Swine/MN/9088-2/ 98 (H3N2)] were propagated and maintained using the Madin-Darby canine kidney (MDCK) cell line (Meguro et al., 1979).

\section{Viral infection}

Cells grown in $10 \mathrm{~cm}$ dishes or T-150 flasks were infected with different SIV subtypes. Each of the SIV subtypes $\left(1 \times 10^{5}\right.$ tissue culture infectious dose $50 ; \mathrm{TCID}_{50}$ ) were inoculated onto the cell monolayer and incubated for $1 \mathrm{~h}$ at $37^{\circ} \mathrm{C}$ to allow cells to be adsorbed by the viruses. PBS was used as a control inoculum. After washing with PBS, cells were grown in Eagle's Minimum Essential Medium (EMEM) supplemented with $2 \%$ FBS for up to $2 \mathrm{~d}$ at $37^{\circ} \mathrm{C}$. Prior- and post-inoculation, the cell monolayer was rinsed twice with $1 \times$ trypsin solution $(1: 1,000$ dilution, GibcoBRL) with SIV.

\section{Analysis of apoptosis}

To determine nuclear genomic DNA fragmentation, one of the hallmarks of apoptosis, genomic DNA was extracted from either mock- or SIV-infected cells using the Wizard genomic DNA purification kit (Promega) according to the protocols of the manufacturer. Genomic DNA $(5 \mu \mathrm{g})$ was subjected to electrophoresis (1.8\% agarose gel) and visualized by staining with ethidium bromide. To examine the apoptotic cell population infected with either mock or SIV, annexin V-stained cell populations were analyzed using the Annexin-V-FLUOS staining kit (Roche) and the FACScan flow cytometer (BD BioSciences) after adding propidium iodide (PI) staining to gate out dead cells according to the instructions of the manufacturers.

\section{Isolation of cytoplasmic fractions}

Cytosolic and mitochondrial fractionations were prepared using the Cytochrome c Release Apoptosis Assay Kit (Oncogene) according to the instructions of the manufacturer. In brief, after incubation in $1 \times$ cytosol extraction buffer containing protease inhibitor, cells were subjected to homogenization with tissue grinder. The efficiency of homogenization was checked by microscopy. When the nuclei $(70-80 \%)$ were observed to have lost their tiny rings, homo- 
A

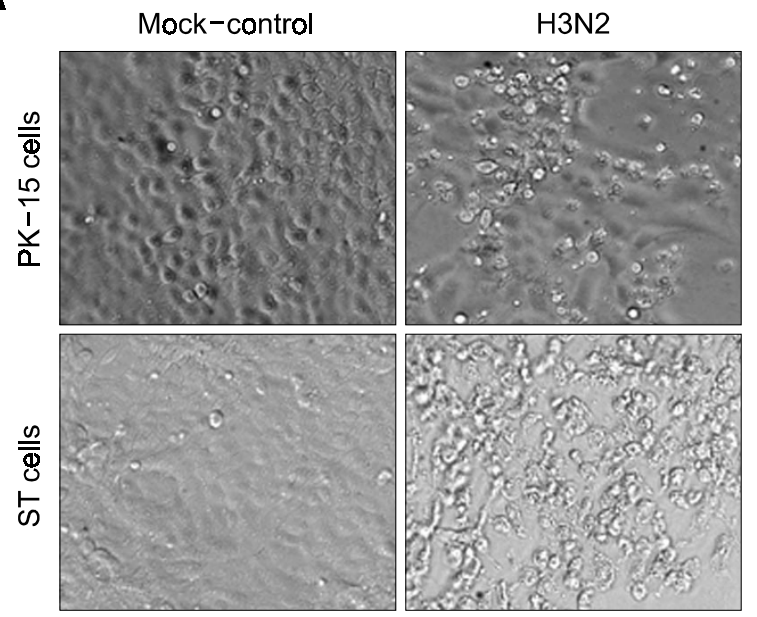

B

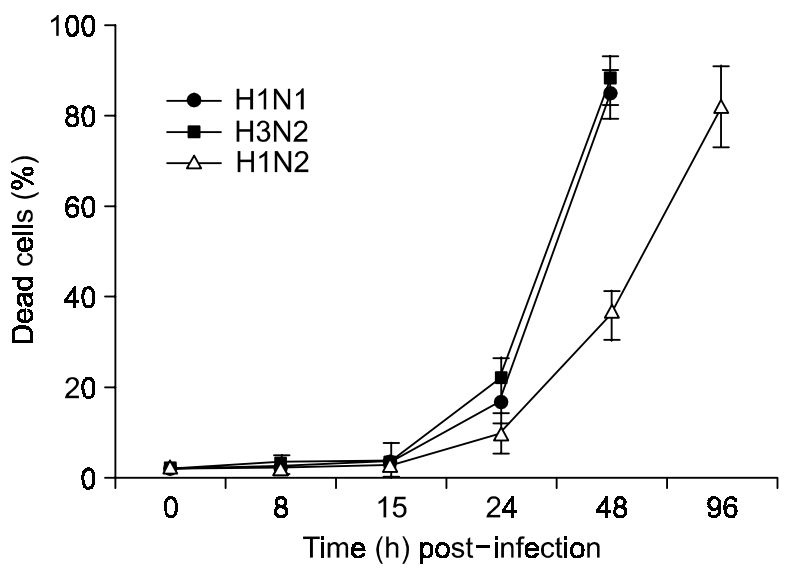

Figure 1. Infection of swine influenza virus (SIV) in porcine cell lines. (A) Representative photographs showing porcine kidney (PK-15) and swine testicle (ST) cell lines infected with either mock-control or H3N2 subtype of swine influenza virus (SIV). (B) Cell death rate (\%) of PK-15 cells infected with the H1N1, H3N2, and H1N2 subtypes of SIV at different time points post-infection. The similar results were obtained from three identical experiments that were independently carried out.

genates were transferred to a microcentrifuge tube $(1.5 \mathrm{ml})$ and centrifuged at $700 \times \mathrm{g}$ for $10 \mathrm{~min}$ at $4^{\circ} \mathrm{C}$. The supernatants were then transferred to a fresh microcentrifuge tube and centrifuged at 10,000 $\times g$ for $30 \mathrm{~min}$ at $4{ }^{\circ} \mathrm{C}$. In this step, supernatants were collected as cytosolic fractions; pellets were resuspended in $0.1 \mathrm{ml}$ mitochondrial extraction buffer and were then saved as mitochondrial fractions.

\section{Immunoprecipitation and Western blot analysis}

Immunoprecipitation assay was conducted using whole cell lysate $(200 \mu \mathrm{g})$, anti-cytochrome c antibody (Oncogene), and Protein A PLUS-Agarose (Santa Cruz Biotechnology). The whole cell extracts, cytosolic fractions, and immunoprecipitates were mixed with sample buffer $(0.5 \mathrm{M}$ Tris- $\mathrm{Cl}, \mathrm{pH} 6.8,10 \%$ SDS, 2-mercaptoethanol, $0.05 \%$ bromphenol blue). The protein samples were boiled at $100^{\circ} \mathrm{C}$ for 4 min and were then cooled on ice. Fifteen microgram of each of the denatured protein samples was separated by 8 or $10 \%$ SDS-polyacrylamide gel and then transferred to a PVDF membrane (Millipore). The membranes were blocked with $5 \%$ nonfat milk and incubated with anti-Bcl-2 (Santa Cruz Biotechnology), anti-Bax (Santa Cruz Biotechnology), anticytochrome c (Santa Cruz Biotechnology), antiApaf-1 (Santa Cruz Biotechnology), anti-caspase-9 (Santa Cruz Biotechnology), anti-TAJ (Oncogene), anti-c-Jun (Oncogene), and anti- $\beta$-actin (Santa Cruz Biotechnology) antibodies. Membranes were then incubated with horseradish peroxidase-conjugated anti-rabbit and anti-mouse IgG antibodies (Pierce) and visualized using SuperSignal West Pico Che- miluminescent Substrate (Pierce).

\section{Results}

\section{Apoptosis of the various cells infected with SIV}

Porcine kidney epithelial (PK-15) cells and swine testicle (ST) cells were infected with one of the three different subtypes (H1N1, H1N2, and H3N2) of SIV. Cytopathic effects (CPE) were not observed until $15 \mathrm{~h}$ post-infection and were less than $30 \%$ at 24 h post-infection with $\mathrm{H} 1 \mathrm{~N} 1$ and $\mathrm{H} 3 \mathrm{~N} 2$ subtypes. H1N2- infected cells, however, did not show CPE until $36 \mathrm{~h}$ post-infection. Maximum CPEs in the PK-15 and ST cells were observed at $48 \mathrm{~h}$ post-infection with $\mathrm{H} 1 \mathrm{~N} 1$ and $\mathrm{H} 3 \mathrm{~N} 2$, and at $96 \mathrm{~h}$ post-infection with the H1N2 SIV subtype (Figure $1 \mathrm{~A}$ and $\mathrm{B}$ ).

We performed annexin- $\mathrm{V}$ and propidium iodide (PI) staining to determine whether CPE occur as a result of apoptosis by SIV replication in the PK-15, ST and HeLa cells. As shown in Figure 2A, high levels of apoptotic cell death were observed in live virus-infected PK-15, ST and HeLa cells, but were not seen in mock-infected and UV-irradiated (dead) virus-infected counterpart cells. Genomic DNA fragmentations were also observed in the PK-15 and ST cells infected with live viruses, but not observed in mock-infected and UV-irradiated virus-infected counterpart cells (Figure 2B). These results suggest that SIV-induced apoptosis in PK-15 and ST cells might be accompanied by replication of live virus, not by just attachment of the virus to cells, as judged by the lack of CPE and apoptotic effects in the cells inoculated with UV-irradiated (infection-com- 
A

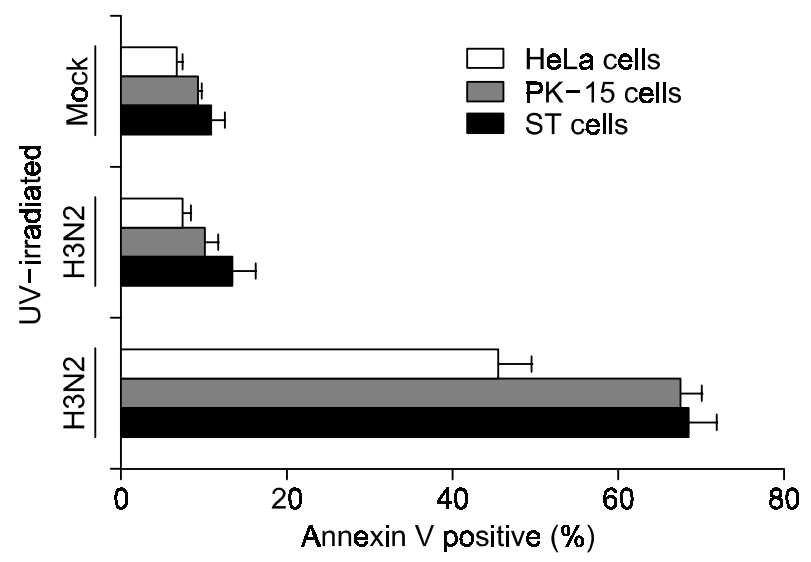

B

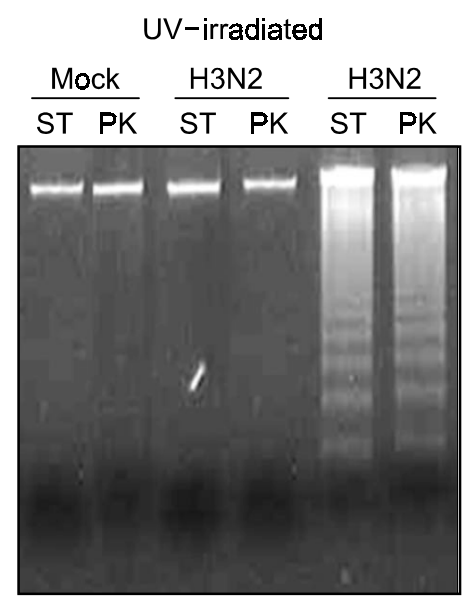

Figure 2. Apoptosis analysis of cells infected with the H3N2 subtype of SIV. (A) Annexin V-positive cells (\%) of HeLa, swine testicle (ST), and PK-15 cells infected with mock-control, UV-irradiated H3N2 subtype (dead virus) or non-treated H3N2 subtype (live virus) of SIV. (B) DNA fragmentation analysis of PK-15 and ST cells infected with mock-control, UV-irradiated H3N2 subtype (dead virus) and non-treated H3N2 subtype (live virus) of SIV at $36 \mathrm{~h}$ post-infection.

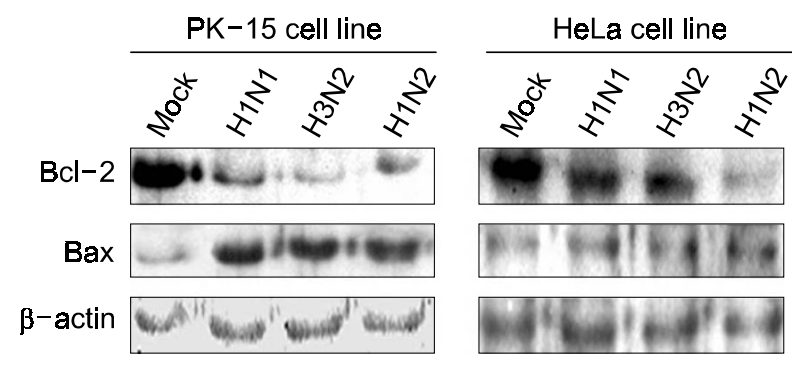

Figure 3. Expression of Bcl-2 and Bax proteins in the SIV-infected cells. PK-15 and HeLa cells were infected with mock-control and different SIV subtypes (H1N1, H3N2 and H1N2). Expression of Bcl-2 and Bax protein was determined by Western blot analysis using anti-Bcl-2, antiBax, and anti- $\beta$-actin (loading control) antibodies.

promised) virus.

\section{Activation of the mitochondrial apoptosis pathway}

Since the Bcl-2 family is associated with apoptosis in a variety of viral infections (Conti et al., 1998; Duncan et al., 1999; Lewis et al., 1999; Mastrangelo et al., 2000; Hong and Wu, 2002), and because expression of $\mathrm{Bcl}-2$, an anti-apoptosis gene, in MDCK cells blocks influenza virus-induced apoptosis and DNA fragmentation (Hinshaw et al., 1994), we determined the expression of $\mathrm{Bcl}-2$ and $\mathrm{Bax}$ proteins to investigate whether members of the $\mathrm{Bcl}-2$ family are implicated in SIV-mediated apoptosis of PK-15 and HeLa cells. As shown in Figure 3, the cellular level of $\mathrm{Bcl}-2$ protein was remarkably downregulated following SIV infection in both cell lines compared to mock-infected control cells at $24 \mathrm{~h}$ post-infection, whereas expression of Bax protein, a pro-apoptosis gene, was upregulated in SIV-infected PK-15 cells, but was not upregulated in SIV-infected HeLa cells. We also determined subcellular localization of Bax protein by Western blot analysis using both cytosolic and mitochondrial fractions of cell lysates. Consistent with expression levels of Bax protein from total cell lysates of mock-infected control and SIV-infected PK-15 cells (Figure 3), Bax protein was found to be increased in the mitochondrial fraction of the SIV-infected PK-15 cells as compared to that of the mock-infected control cells (data not shown). Taken together, these findings indicate that SIV infection in PK-15 cells might provoke cell death by both suppression of anti-apoptosis gene expression and promotion of pro-apoptosis gene expression.

A number of $\mathrm{Bcl}-2$ pro-apoptotic members, such as Bax and Bad, are known to exert their mitochondria-relative pro-apoptotic effects either by interacting with and inhibiting $\mathrm{Bcl}-2$ and $\mathrm{Bcl}-\mathrm{XL}$ or by direct interaction with the mitochondrial membrane, after which they induce the release of cytochrome c from the mitochondria to the cytosol. Hence, we examined whether cytochrome $\mathrm{c}$ is released from mitochondria to cytosol in the SIV-infected cells at $24 \mathrm{~h}$ post-infection. As shown in Figure 4A, the cytosolic release of cytochrome $\mathrm{c}$ was shown to be dramatically increased in the SIV-infected PK-15 cells and slightly increased in the SIV-infected HeLa cells as compared to mock-infected counterpart cells. To further confirm the formation of apoptosomes (multi-protein complexes consisting of cytochrome c, Apaf-1, pro-caspase-9, and ATP), we performed immunoprecipitations using anti-cytochrome $c$ anti- 
A

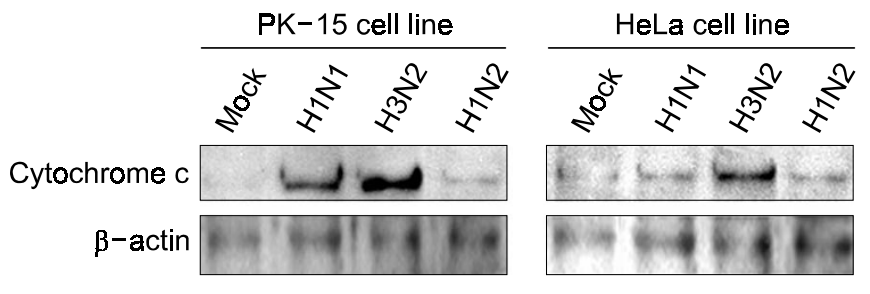

B

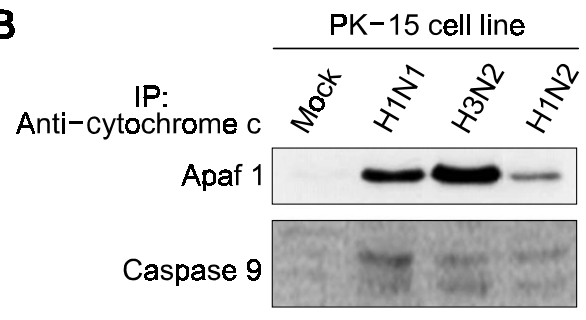

C

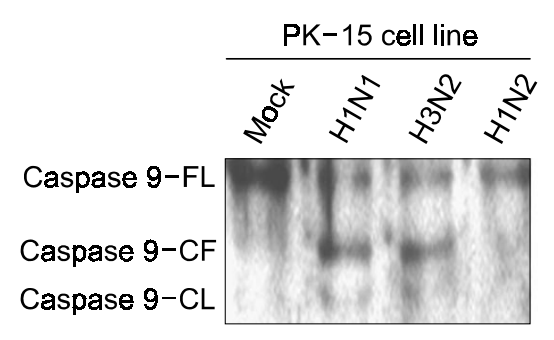

HeLa cell line

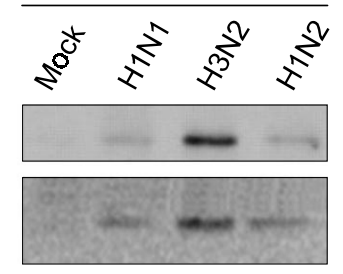

HeLa cell line

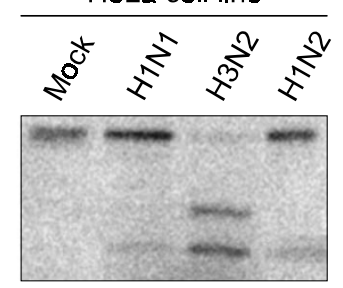

Figure 4. Cytosolic release of cytochrome c, formation of apoptosomes, and activation of caspase-9 in the SIV-infected cells. (A) Cytochrome $c$ released from the mitochondria to the cytosol in the PK-15 and HeLa cells infected with mock-control, H1N1, H3N2, and H1N2 was detected by Western blot analysis. The $\beta$-actin was used as loading control. (B) Apaf-1 and caspase-9 immunoprecipitated by anti-cytochrome $c$ antibody in the PK-15 and HeLa cells infected with mock-control and different SIV subtypes (H1N1, H3N2, and H1N2). (C) Expression levels of the active caspase-9 in the PK-15 and HeLa cells infected with mock-control and different SIV subtypes (H1N1, H3N2, and H1N2). body. The results revealed that more Apaf- 1 and caspase-9 proteins in the SIV-infected PK-15 and HeLa cells were coprecipitated with anti-cytochrome $\mathrm{c}$ antibody than were coprecipitated in mock-infected counterpart cells (Figure 4B). Furthermore, activated caspase-9 (proteolytic fragmented caspase-9) was found to increase in the cytosolic fractions of the SIV-infected PK-15 and HeLa cells, but this was not the case with mock-infected counterpart cells (Figure 4C). Taken together, these results suggest that SIV-mediated apoptosis in the PK-15 and HeLa cells should occur through the intrinsic mitochondriamediated apoptosis pathway.

\section{Activation of stress-associated proteins}

The c-Jun/AP-1 transcriptional factor has been of great interest to researchers because its activity is influenced by oxidative stress and can induce apoptosis (Sawai et al., 1995; Gong et al., 2004; Kim et al., 2004). TAJ, also known as TROY, is a $-46 \mathrm{kDa}$ membrane protein possessing characteristic cysteine-rich motifs in the extracellular domain and a TNF receptor-associated factor (TRAF)-2 binding sequence in the cytoplasmic domain (Eby et al., 2000). TAJ binds a number of different TARF family members and enables the activation of the JNK pathway, most likely via interaction with a TRAF homologue. Since infection of the equine influenza

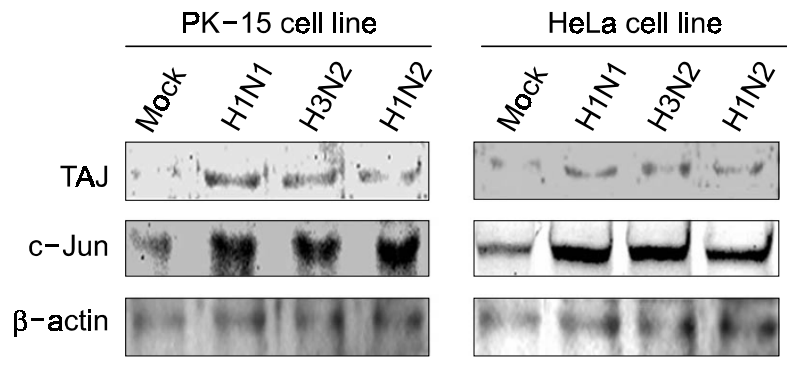

Figure 5. Upregulation of TAJ and C-Jun proteins in the SIV-infected cells. PK-15 and HeLa cells were infected with mock-control and different SIV subtypes (H1N1, H3N2, and H1N2). Expression of TAJ and c-Jun proteins in the PK-15 and HeLa cells infected with mock-control, H1N1, $\mathrm{H} 3 \mathrm{~N} 2$, and H1N2 was determined by Western blot analysis using anti-TAJ, anti-c-Jun, and anti- $\beta$-actin (loading control) antibodies.

virus increased cellular levels of C-Jun/AP-1 protein (Lin et al., 2001), we wondered whether SIV infection activated expression of c-Jun and TAJ. As demonstrated by Western blot, SIV infection was shown to significantly increase cellular levels of c-Jun and TAJ proteins in PK-15 and HeLa cells (Figure 5), suggesting that TAJ and c-Jun upregulated by SIV infection might play a crucial role in SIV-induced stress and apoptosis-signaling pathways. 


\section{Discussion}

Although the death receptor-mediated apoptosis pathway induced by influenza infection has been extensively studied (Takizawa et al., 1995; Wada et al., 1995; Fujimoto et al., 1998), little is known about the potential participation of the mitochondriamediated apoptosis pathway during influenza-induced cell death. Although PK-15 cells are not commonly used for swine influenza virus replication, our results shown in Figure 1 demonstrated that PK-15 cell was shown to fully support SIV replications. Furthermore, we used $\mathrm{PK}-15$ to demonstrate the apoptotic pathway in swine cells because MDCK cells commonly used for SIV culture were originally derived from canine kidney cells. In the present study, we report that SIV infection in the PK-15 and HeLa cells should induce cell death through the mitochondria-mediated apoptosis pathway, as judged by 1) downregulation of anti-apoptotic Bcl-2; 2) upregulation of pro-apoptotic Bax; 3) cytosolic release of cytochrome c from mitochondria; and 4) activation of caspase-9, all of which are known to be implicated in mitochondria-mediated apoptosis. We used three different swine influenza virus subtypes ( $\mathrm{H} 1 \mathrm{~N} 1$, $\mathrm{H} 1 \mathrm{~N} 2$, and H3N2) to determine whether they exert similar cellular effects in terms of apoptosis. The H1N2 subtype showed relatively delayed CPE in the infected cells as compared to $\mathrm{H} 1 \mathrm{~N} 1$ and $\mathrm{H} 3 \mathrm{~N} 2$ subtypes. The delayed CPE in the H1N2 infected cells might result from decrease of cell death rate, cytochrome c release and apoptosome formation as compared to those of $\mathrm{H} 1 \mathrm{~N} 1$ and $\mathrm{H} 3 \mathrm{~N} 2$ infected cells. Interestingly, H1N1 and H1N2 subtypes did not show obvious CPE in the HeLa cells (data not shown) and failed to induce cytosolic release of cytochrome c and formation of apoptosomes (Figure 4), whereas H3N2 subtype did. These results suggest that virusinduced cell death might be differentially regulated depending upon cell type and viral factors.

Although upregulation of c-Jun and TAJ in the SIV-infected cells indicates that SIV-induced apoptosis might be partially activated by the stress-signaling pathway, the stress-signaling pathway appears not to be sufficient for inducing SIV-mediated apoptosis, because TAJ and c-Jun were shown to be markedly increased in the H1N1 and H1N2-infected HeLa cells which did not show apoptotic cell death. It is plausible that SIV-mediated apoptosis might be regulated by both receptor- and mitochondrial-mediated cell death.

\section{Acknowledgement}

This work was supported by a BioGreen 21 grant and Ministry of Education and Human Resources Development in Korea (2005) awarded to Dr. Hyunggee Kim.

\section{References}

Choi YK, Goyal SM, Joo HS. Prevalence of swine influenza virus subtypes on swine farms in the United States. Arch Virol 2002;147:1209-20

Conti L, Rainaldi G, Matarrese P, Varano B, Rivabene R, Columba S, Sato A, Belardelli F, Malorni W, Gessani S. The HIV-1 vpr protein acts as a negative regulator of apoptosis in a human lymphoblastoid $T$ cell line: possible implications for the pathogenesis of AIDS. J Exp Med 1998;187:403-13

Duncan R, Muller J, Lee N, Esmaili A, Nakhasi HL. Rubella virus-induced apoptosis varies among cell lines and is modulated by Bcl-XL and caspase inhibitors. Virology 1999;255: 117-28

Eby MT, Jasmin A, Kumar A, Sharma K, Chaudhary PM. TAJ, a novel member of the tumor necrosis factor receptor family, activates the c-Jun N-terminal kinase pathway and mediates caspase-independent cell death. J Biol Chem 2000;275:15336-42

Fujimoto I, Takizawa T, Ohba Y, Nakanishi Y. Co-expression of Fas and Fas-ligand on the surface of influenza virus-infected cells. Cell Death Differ 1998;5:426-31

Gong X, Wang M, Wu Z, Tashiro S, Onodera S, Ikejima T. Pseudolaric acid B induces apoptosis via activation of c-Jun N-terminal kinase and caspase-3 in HeLa cells. Exp Mol Med 2004;36:551-6

Hengartner MO. The biochemistry of apoptosis. Nature 2000;407:770-6

Hinshaw VS, Olsen CW, Dybdahl-Sissoko N, Evans D. Apoptosis: a mechanism of cell killing by influenza $A$ and $B$ viruses. J Virol 1994;68:3667-73

Hong JR, Wu JL. Induction of apoptotic death in cells via Bad gene expression by infectious pancreatic necrosis virus infection. Cell Death Differ 2002;9:113-24

Jacobson MD, Weil M, Raff MC. Programmed cell death in animal development. Cell 1997;88:347-54

Kim YS, Jhon DY, Lee KY. Involvement of ROS and JNK1 in selenite-induced apoptosis in Chang liver cells. Exp Mol Med 2004;36:157-64

Kumar S. Mechanisms mediating caspase activation in cell death. Cell Death Differ 1999;6:1060-6

Lewis J, Oyler GA, Ueno K, Fannjiang YR, Chau BN, Vornov J, Korsmeyer SJ, Zou S, Hardwick JM. Inhibition of virus-induced neuronal apoptosis by Bax. Nat Med 1999;5:832-5

Lin C, Zimmer SG, Lu Z, Holland RE Jr, Dong Q, Chambers TM. The involvement of a stress-activated pathway in equine influenza virus-mediated apoptosis. Virology 2001;287:202-13

Ludwig S, Pleschka S, Wolff T. A fatal relationship--influenza virus interactions with the host cell. Viral Immunol 1999;12: 175-96

Majno G, Joris I. Apoptosis, oncosis, and necrosis. An overview of cell death. Am J Pathol 1995;146:3-15

Mastrangelo AJ, Hardwick JM, Bex F, Betenbaugh MJ. Part I. BCl-2 and Bcl-x (L) limit apoptosis upon infection with alphavirus vectors. Biotechnol Bioeng 2000;67:544-54 
Meguro $\mathrm{H}$, Bryant JD, Torrence AE, Wright PF. Canine kidney cell line for isolation of respiratory viruses. J Clin Microbiol 1979;9:175-9

Mori I, Komatsu T, Takeuchi K, Nakakuki K, Sudo M, Kimura Y. In vivo induction of apoptosis by influenza virus. J Gen Virol 1995;76:2869-73

Morris SJ, Price GE, Barnett JM, Hiscox SA, Smith H, Sweet C. Role of neuraminidase in influenza virus-induced apoptosis. J Gen Virol 1999;80:137-46

Olsen CW, Kehren JC, Dybdahl-Sissoko NR, Hinshaw VS. bcl-2 alters influenza virus yield, spread, and hemagglutinin glycosylation. J Virol 1996;70:663-6

Rich T, Allen RL, Wyllie AH. Defying death after DNA damage. Nature 2000;407:777-83

Roulston A, Marcellus RC, Branton PE. Viruses and apoptosis. Annu Rev Microbiol 1999;53:577-628

Sawai H, Okazaki T, Yamamoto H, Okano H, Takeda $\mathrm{Y}$, Tashima M, Sawada H, Okuma M, Ishikura H, Umehara H, Domae N. Requirement of AP-1 for ceramide-induced apoptosis in human leukemia HL-60 cells. J Biol Chem 1995; 270:27326-31
Schultz-Cherry S, Dybdahl-Sissoko N, Neumann G, Kawaoka $\mathrm{Y}$, Hinshaw VS. Influenza virus ns1 protein induces apoptosis in cultured cells. J Virol 2001;75:7875-81

Takizawa T, Fukuda R, Miyawaki T, Ohashi K, Nakanishi Y. Activation of the apoptotic Fas antigen-encoding gene upon influenza virus infection involving spontaneously produced beta-interferon. Virology 1995;209:288-96

Takizawa T, Tatematsu C, Ohashi K, Nakanishi Y. Recruitment of apoptotic cysteine proteases (caspases) in influenza virus-induced cell death. Microbiol Immunol 1999;43:245-52

Teodoro JG, Branton PE. Regulation of apoptosis by viral gene products. J Virol 1997;71:1739-46

Wada N, Matsumura M, Ohba Y, Kobayashi N, Takizawa T, Nakanishi Y. Transcription stimulation of the Fas-encoding gene by nuclear factor for interleukin- 6 expression upon influenza virus infection. J Biol Chem 1995;270:18007-12

Young LS, Dawson CW, Eliopoulos AG. Viruses and apoptosis. Br Med Bull 1997;53:509-21

Zheng TS, Schlosser SF, Dao T, Hingorani R, Crispe IN, Boyer JL, Flavell RA. Caspase-3 controls both cytoplasmic and nuclear events associated with Fas-mediated apoptosis in vivo. Proc Natl Acad Sci USA 1998;95:13618-23 